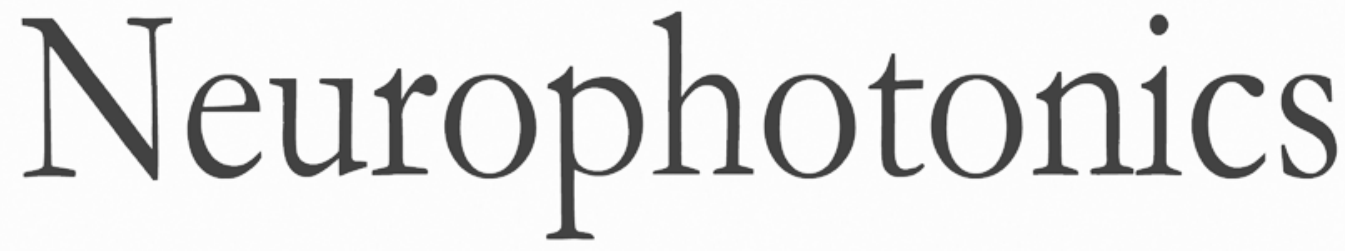

\title{
Evaluation of evoked responses to pulse-matched high frequency and intermittent theta burst transcranial magnetic stimulation using simultaneous functional near-infrared spectroscopy
}

\author{
Adrian Curtin \\ Junfeng Sun \\ Hasan Ayaz \\ Zhenying Qian \\ Banu Onaral \\ Jijun Wang \\ Shanbao Tong
}




\title{
Evaluation of evoked responses to pulse-matched high frequency and intermittent theta burst transcranial magnetic stimulation using simultaneous functional near-infrared spectroscopy
}

\author{
Adrian Curtin, ${ }^{\mathrm{a}, \mathrm{b}}$ Junfeng Sun, ${ }^{\mathrm{a}}$ Hasan Ayaz, ${ }^{\mathrm{b}, \mathrm{c}, \mathrm{d}, \star}$ Zhenying Qian, ${ }^{\mathrm{e}}$ Banu Onaral, ${ }^{\mathrm{b}}$ Jijun Wang, ${ }^{\mathrm{e}, \star}$ and \\ Shanbao Tong ${ }^{\mathrm{a}, *}$ \\ aShanghai Jiao Tong University, School of Biomedical Engineering, Shanghai, China \\ brexel University, School of Biomedical Engineering, Science, and Health Systems, Philadelphia, Pennsylvania, United States \\ 'University of Pennsylvania, Department of Family and Community Health, Philadelphia, Pennsylvania, United States \\ ${ }^{d}$ Children's Hospital of Philadelphia, The Division of General Pediatrics, Philadelphia, Pennsylvania, United States \\ e'Shanghai Jiao Tong University, School of Medicine, Shanghai Mental Health Center, Shanghai, China
}

\begin{abstract}
Transcranial magnetic stimulation (TMS) is a noninvasive method used to excite or inhibit cortical activity for experimental, diagnostic, and therapeutic interventions. However, nonmotor regions of the brain targeted in TMS therapies, such as the dorsolateral prefrontal cortex (DLPFC), offer no extrinsic response to stimulation, resulting in a need for a practical method for the evaluation of treatment. We sought to determine the capability of a continuous-wave light emitting diodes (LED)-based functional near-infrared spectroscopy (fNIRS) system to measure evoked cortical hemoglobin changes in the DLPFC during the simultaneous application of TMS to the left-DLPFC under brief stimulation paradigms used in the clinic. Seventeen healthy participants received short TMS trains at F3 in four different stimulation conditions (single pulse, high frequency, intermittent theta burst, and sham) while adjacent fNIRS measurements were recorded. Ten 2-s trains of each stimulation type were delivered with an intertrial interval of $40 \mathrm{~s}$. Results indicated that high-frequency stimulation produces a larger and more evident response than other measured conditions. These findings show that a continuous-wave LED-based fNIRS system can be used to measure TMS-evoked responses and that future TMS applications can benefit from concurrent assessment of localized cortical activation changes. ๑ 2017 Society of Photo-Optical Instrumentation Engineers (SPIE) [DOI: 10.1117/1.NPh.4.4.041405]
\end{abstract}

Keywords: functional near-infrared spectroscopy; repetitive transcranial magnetic stimulation; intermittent theta burst stimulation; mental health; prefrontal cortex; neurostimulation.

Paper 17077SSRR received Apr. 1, 2017; accepted for publication Jul. 7, 2017; published online Aug. 17, 2017.

\section{Introduction}

Repetitive transcranial magnetic stimulation (rTMS) is a noninvasive brain stimulation method that can be used to excite or inhibit cortical activity via electromagnetically induced currents. TMS has been used as a tool for experimental brain research $^{1}$ as well as a valuable nonpharmacological therapeutic technique in major depressive disorder (MDD) with developing applications in Schizophrenia and a variety of other mental disorders. ${ }^{2}$ When used in MDD treatment, TMS frequently targets the left-dorsolateral prefrontal cortex (DLPFC) in order to activate the underlying cortex using patterns that induce longterm potentiation (LTP), such as conventional high-frequency stimulation $(>5 \mathrm{~Hz})$ and the neurobiologically-inspired intermittent theta burst stimulation (iTBS). iTBS is a TMS paradigm adapted from animal studies by Huang et al. ${ }^{3}$ based on observations that short trains of $50 \mathrm{~Hz}$ stimulation at the theta rhythm of $5 \mathrm{~Hz}$ reliably induced LTP in the motor cortex and hippocampus, mimicking the coupling of gamma and theta rhythms. iTBS stimulation trains are of particular interest in the clinical

\footnotetext{
*Address all correspondence to: Hasan Ayaz, E-mail: hasan.ayaz @ drexel.edu Jijun Wang, E-mail: jijunwang27@gmail.com; Shanbao Tong, E-mail: shanbao.tong@gmail.com
}

community because their use translates to a shorter therapeutic duration at lower intensities ( $80 \%$ to $90 \%$ RMT versus $>110 \%$ RMT $)^{4,5}$ than conventional suprathreshold high-frequency rTMS protocols. Although both techniques have shown promise in the treatment of medication-resistant MDD,${ }^{6}$ not all patients respond successfully to TMS therapies. The selection of appropriate parameters, such as the specific patterns used, may be an important step in improving clinical response to therapy. ${ }^{7}$ However, determination of these parameters is made more difficult by the fact that areas such as the DLPFC produce no overt muscular response to stimulation. Therefore, physicians can evaluate rTMS only in terms of patient tolerance to treatment and, after a number of rTMS sessions, changes to subjective clinical measures.

Measuring neural activity, such as the evoked hemodynamic responses associated with stimulation, may help identify best treatment practices, identify responders to therapy, and eventually help address problems related to intersubject heterogeneity through an individualized approach to therapy. ${ }^{8}$ Neurohemodynamic measures such as regional cerebral blood flow (rCBF) of positron emission tomography ${ }^{9,10}$ and the blood oxygen

$2329-423 X / 2017 / \$ 25.00$ (C) 2017 SPIE 
level-dependent (BOLD) response of $\mathrm{fMRI}^{11,12}$ have been shown to be differentially sensitive to TMS parameters and in some cases reported to predict treatment response. ${ }^{13}$ However, rTMS must occur over a number of sessions and these systems cannot be practically scaled for use in clinical settings.

Functional near infrared spectroscopy (fNIRS) is an optical technique that uses specific wavelengths of light within the region of 700 to $900 \mathrm{~nm}$ to measure concentrations of oxygenated $[\mathrm{HbO}]$ and deoxygenated $[\mathrm{Hb}]$ hemoglobin in the cerebral cortex. ${ }^{14}$ fNIRS is a promising method for concurrent TMS imaging as the technique relies on measurement of light that is not directly perturbed by magnetic flux, has comparable spatial resolution to the foci of TMS coils ${ }^{15}(\mathrm{~cm})$ and has the temporal resolution to capture evoked hemodynamic responses with higher precision than those offered by interleaved-fMRI. ${ }^{16}$ Additionally, the technique can scale well for clinical applications as it is noninvasive, easily applied, capable of long-term measurements, and can be made more cost-effective through the use of probes containing light emitting diodes (LED)-based light sources. In a recent study, we have demonstrated simultaneous fNIRS and transcranial direct current stimulation of the anterior prefrontal cortex (PFC) during cognitive tasks, highlighting the complementary qualities of fNIRS for neurostimulation. ${ }^{17}$

fNIRS has been used previously in conjunction with TMS, but only a few studies have examined stimulation in the DLPFC, the area most typically used in the clinic. While some studies conducted have examined results in the motor cortex, conflicting findings suggest that TMS-evoked responses measured in this region cannot necessarily be considered as stereotypical of other regions. In the motor cortex (M1), single pulses of TMS delivered at rest using subthreshold levels have been reported to decrease $[\mathrm{Hb}]^{18}$ during stimulation or increase $[\mathrm{HbO}],{ }^{19,20}$ both with conflicting effects on total hemoglobin $[\mathrm{HbT}]$, whereas in the PFC, a decrease in [HbO] over the DLPFC was observed only at a high suprathreshold level $(130 \%),{ }^{21,22}$ an intensity not normally used during treatment. One study has examined high-frequency stimulation in the DLPFC, but using only $5 \mathrm{~Hz}$, even though the most common frequencies used in the clinic range from 10 to $20 \mathrm{~Hz}$. Additionally, alternative LTPinducing paradigms to HF stimulation such as iTBS have not been examined at all in the DLPFC. As such, the expected responses to TMS stimulation trains in healthy subjects cannot be described as fully characterized.

In this study, we investigated the evoked response to short trains of four TMS stimulation conditions: high frequency, single pulse, intermittent theta burst, and sham, using fNIRS sensors simultaneously. To our knowledge, this is the first concurrent fNIRS-TMS multimodal investigation to assess iTBS in the DLPFC and the first usage of an LED-based fNIRS system in the measurement of any TMS-evoked responses.

\section{Methods}

\subsection{Subject Demographics}

Seventeen healthy right-handed volunteers ( 9 male, 8 female; age: mean 26.6, S.D. 2.6) were recruited from the local community and provided informed consent prior to participation in this study. Participants self-reported no history of mental illness or drug abuse, right-handedness, and were compensated for their time. Demographic information is reported in Table 1. The study took place at the Shanghai Mental Health Center and consisted of two sessions per day over two days (four sessions
Table 1 Subject demographics.

\begin{tabular}{|c|c|c|c|}
\hline Subject & Gender & Age & RMT (\%) \\
\hline 1 & M & 28 & 39 \\
\hline 2 & $\mathrm{~F}$ & 28 & 43 \\
\hline 3 & $\mathrm{~F}$ & 24 & 41 \\
\hline 4 & $\mathrm{~F}$ & 25 & 30 \\
\hline 5 & M & 34 & 47 \\
\hline 6 & $\mathrm{~F}$ & 25 & 34 \\
\hline 7 & $\mathrm{~F}$ & 25 & 38 \\
\hline 8 & $\mathrm{~F}$ & 27 & 27 \\
\hline 9 & M & 23 & 34 \\
\hline 10 & M & 27 & 40 \\
\hline 11 & $\mathrm{~F}$ & 26 & 41 \\
\hline 12 & M & 25 & 32 \\
\hline 13 & $M$ & 28 & 43 \\
\hline 14 & M & 27 & 34 \\
\hline 15 & M & 25 & 26 \\
\hline 16 & $F$ & 25 & 34 \\
\hline 17 & $\mathrm{~F}$ & 30 & 39 \\
\hline
\end{tabular}

total), separated by a minimum of $1 \mathrm{~h}$ (mean $3.3 \mathrm{~h}$, S.D. $47 \mathrm{~min}$ ) to reduce the effect of carry-over from TMS stimulation types. Procedures were approved according to the Ethics Committee of the Shanghai Mental Health Center.

\subsection{Experimental Setup}

After obtaining written consent, participants had their resting motor threshold (RMT: mean 36.6\%, S.D. 5.9) estimated. rTMS stimulation was performed using a figure-of-eight coil (MCF-B65) and the MagPro system (MagVenture, Denmark). The stimulation site was then identified according to placement on F3 as identified by the international 10-20 system. This location has been reported to be a more accurate estimation of the location of the left DLPFC when compared to the $5-\mathrm{cm}$ rule. ${ }^{23}$ The fNIR1100 sensor (fNIR Devices LLC, Potomac, Maryland) was centered in line with FP1 and FP2 of the international 10-20 system on the subject's forehead and the sensor settings were adjusted to receive the highest signal levels without saturation, accounting for subject dependencies and lighting conditions. For data acquisition and visualization, COBI Studio software was used. ${ }^{24}$ After calibration of the sensor, fNIRS biomarkers were recorded continuously at $2 \mathrm{~Hz}$ over the Frontopolar region of the DLPFC (BA10). Placement of the fNIRS sensor was just dorsal to the stimulation location and the TMS device was typically adjacent but not overlapping with optode 3 on the sensor (as shown in Fig. 1). 
(a)

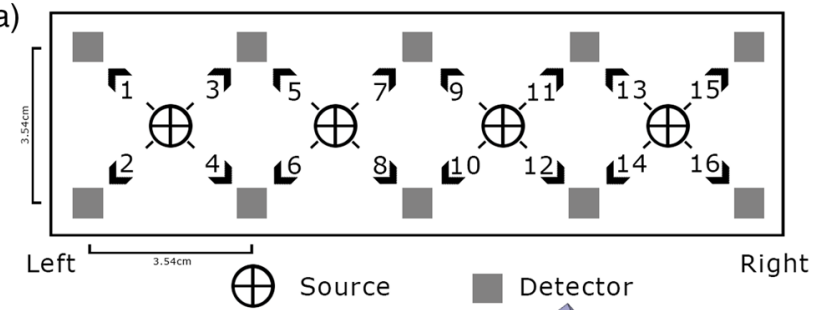

(b)

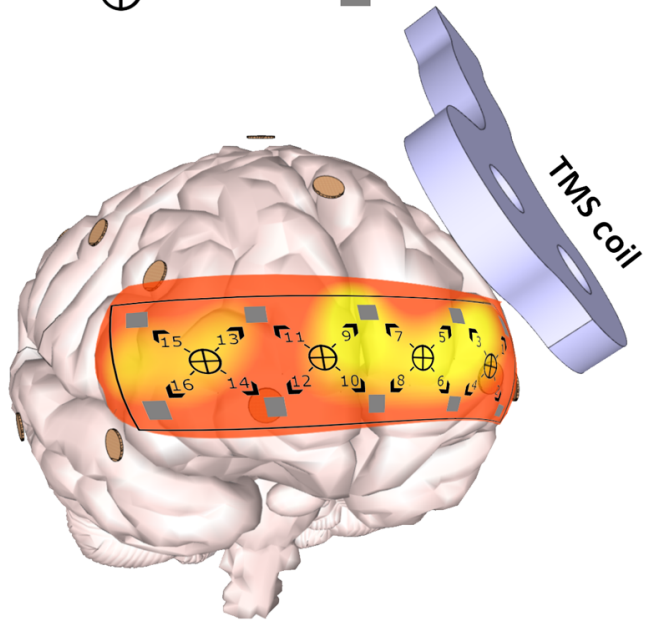

Fig. 1 (a) Optode arrangement for fNIR1100 sensor and (b) TMS coil placement at $\mathrm{F} 3$ relative to measured area in BA10 with approximate optode positions marked for reference.

\subsection{Repetitive Transcranial Magnetic Stimulation Procedure}

In order to examine the prefrontal response to different rTMS paradigms, subjects were assigned randomly to one of four stimulation orders and these subgroups were used to account for the effects of order on TMS response. Subjects were measured over the course of four sessions across two days (2 sessions/day). Each session consisted of 10 trials of a single stimulation pattern and sessions were separated by at least $1 \mathrm{~h}$ to wash out poststimulation effects. TMS stimulation was given in an individual train lasting $2 \mathrm{~s}$ with $40 \mathrm{~s}$ between trials (Fig. 2). During stimulation, fNIRS was recorded online to determine the hemodynamic time course of TMS-evoked responses over the frontopolar region. TMS stimulation was synchronized with a fNIRS recording computer via the use of the RS232 output integrated into the MagPro system.

In all trials, TMS stimulation was delivered over the F3 area as identified by the international $10-20$ system. Single pulse stimulation was delivered at $110 \%$ RMT, whereas highfrequency stimulation was given with 30 pulses delivered at
$110 \%$ RMT at $15 \mathrm{~Hz}$. Theta burst stimulation was delivered at $90 \%$ RMT with triple $50-\mathrm{Hz}$ pulses spaced at $5 \mathrm{~Hz}$ for a total of 30 pulses. Suprathreshold high-frequency stimulation was used according to findings of greater clinical efficacy with the technique ${ }^{25,26}$ and absence of a local BOLD response to subthreshold HF stimulation in fMRI studies. ${ }^{27-29}$ Use of subthreshold theta burst stimulation was inline with current safety recommendation ${ }^{30}$ following reported risk of seizure with $100 \%$ RMT TBS delivery. Sham stimulation, used as control, was produced by reversing the coil and using the high-frequency stimulation condition to produce the sounds and vibrations of stimulation without the corresponding flux. No adverse effects due to TMS or online recording were reported by any subject during this study.

\subsection{Functional Near-Infrared Spectroscopy Signal Processing}

Raw light intensity data were collected for 48 channels corresponding to 16 optodes ( 3 channels per optode: 2 wavelengths +1 ambient light intensity reference) at $2 \mathrm{~Hz}$ from the PFC using the fNIR1100 imager. Data were screened for motion artifacts and saturated signals. Motion artifacts were rejected using the sliding motion artifact rejection algorithm ${ }^{31}$ and a bandpass filter was used to reduce high-frequency noise and account for global trends in the raw-light intensity using cutoffs of 0.01 and $0.25 \mathrm{~Hz}$. The 10th stimulation trial was dropped in all comparisons due to subject motion associated with completion of the session. The physiological measurements in terms of $\Delta[\mathrm{HbO}]$ and $\Delta[\mathrm{Hb}]$ were then calculated using the modified Beer-Lambert law ${ }^{32}$ and corrected values of activation were estimated using the correlation-based signal improvement (CBSI) algorithm. ${ }^{33}$ Measured values were reported as the average change in hemoglobin concentration from the time of initial stimulation. Extracted measures of CBSI-corrected $\mathrm{HbO}$ are reported as [HbO].

\subsection{Statistical Analysis}

Data analysis was conducted using $\mathrm{R}$ (version 3.2.2) and MATLAB (version R2016A). A linear mixed-effects (LME) model for the fixed effect of stimulation type was evaluated for each individual optode. Subject means were allowed to vary around an individualized intercept across trials with stimulation type as a within subject factor. Statistical significance of model fixed effects was assessed using the Satterthwaite approximation for degrees of freedom and significance was corrected for family-wise error rate using the false discovery rate $^{34}$ across all optodes with $q=0.1$.

LME models are typically preferred to classical analysis techniques due to their better ability to handle unbalanced

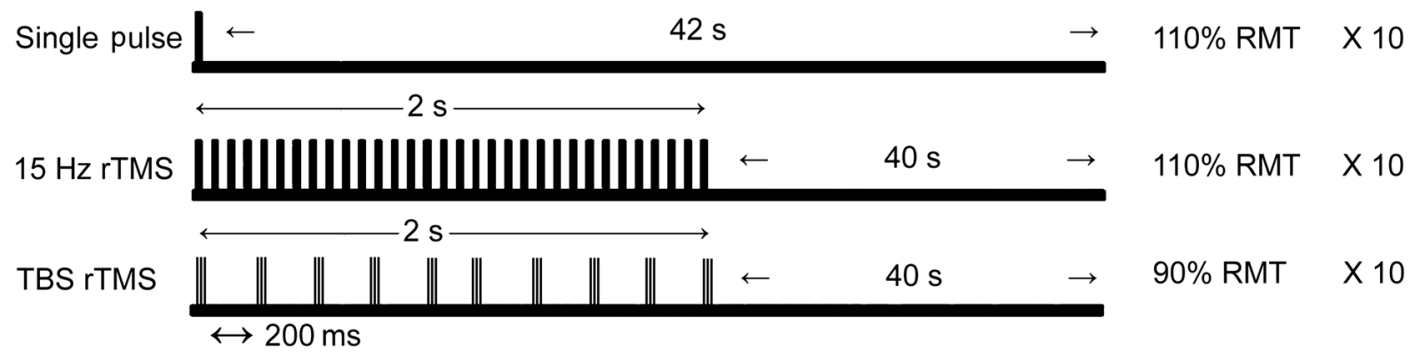

Fig. 2 TMS stimulation trial timing. 


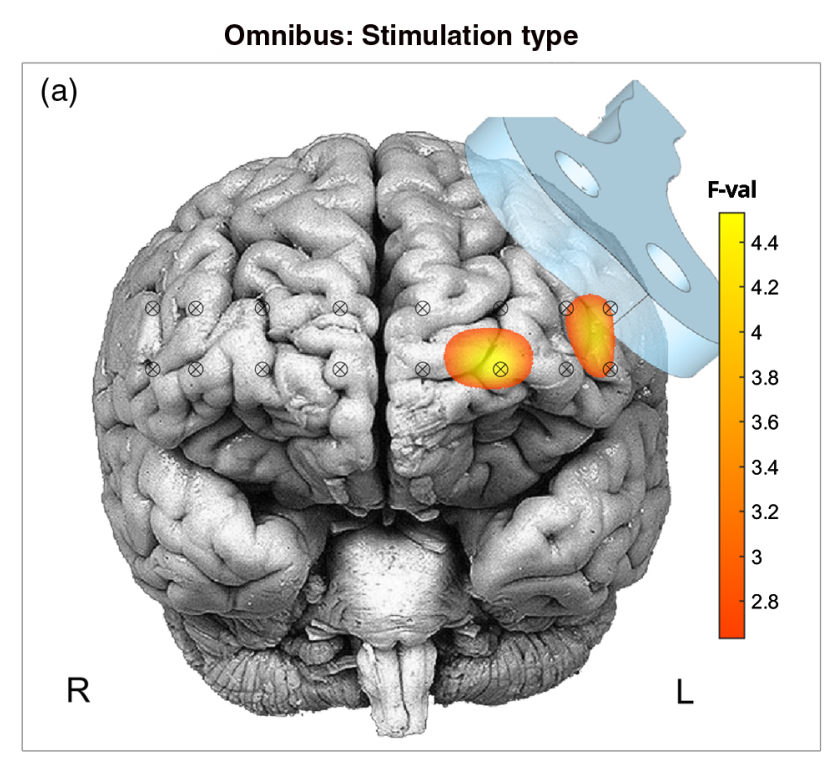

(b)

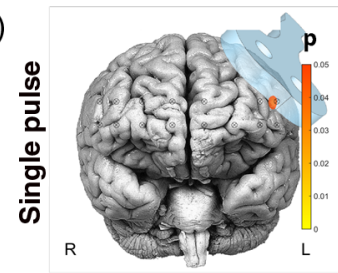

(c)

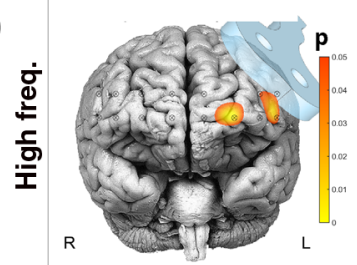

(d)

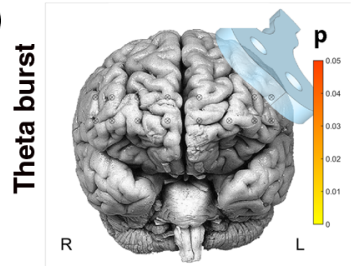

Fig. 3 Parametric mapping comparisons for all subjects. (a) Omnibus test for main effect of stimulus type across all sessions; exploratory post hoc comparisons of all conditions, (b) single pulse versus sham, (c) high frequency versus sham, and (d) theta burst versus sham.

data, intersubject variability and are generally better suited for interpretation of neuroimaging data. ${ }^{35}$ Redundant analysis was conducted using repeated measures analysis of variance (ANOVA) to allow simpler interpretation of the results.

Post hoc comparisons were adjusted for multiplicity using the joint normal distribution of the linear function. Significance maps of each stimulation condition with sham are presented for exploratory purposes. Optodes that survived an optode-wise FDR-correction for the omnibus test are presented temporally. Time course plots were generated from the average change of each second from the average immediately surrounding stimulation (i.e., from -1 to $1 \mathrm{~s}$ ). Plots show the mean value of each second and the standard error of the mean for each time point. Significant differences as determined by uncorrected $t$-tests are marked at different time points for illustration.

\section{Results}

The grand-averaged waveforms indicated strongest increases in [HbO] for optodes $1,2,3$, and 6 for the high-frequency condition relative to sham. Changes appeared from about $4 \mathrm{~s}$ after the onset of stimulation and peaked between 8 and $15 \mathrm{~s}$ varying among individual optodes. Single pulse stimulation appeared to induce changes in optode 1 , peaking $4 \mathrm{~s}$ after stimulation. Theta pulse stimulation appeared to induce marginal changes in optode 6 , peaking $15 \mathrm{~s}$ following stimulation.

On the basis of previous studies ${ }^{21,36}$ and inference from the hemodynamic response time course, we expected changes to begin to evolve $\sim 4$ to $6 \mathrm{~s}$ after the stimulation. We qualitatively observed that hemodynamic changes associated with stimulation appeared to persist until $\sim 15 \mathrm{~s}$ after the stimulation. For the purposes of statistical testing, we selected a window from 10 to $15 \mathrm{~s}$ following the onset of the stimulation as our time range of interest for further evaluation. Evaluation with the
LME model omnibus revealed a significant main effect for stimulation type across optodes 2 and $6(p<0.05)$ for $[\mathrm{HbO}]$. FDR correction was applied to omnibus tests and significant main effects remained for optode $6[q=0.0629, F$ (4.53, $\mathrm{d} F=350.48), p=0.0039]$. The FDR corrected omnibus test is presented parametrically in Fig. 3(a). We also used repeated measures ANOVA as a classical statistics comparison and again found that optode 6 was significant following FDR correction for stimulation type as a within-subject term $[q=0.0380$, $F(5.56, \mathrm{~d} F=3), p=0.0024]$. No main effect for stimulation type on $[\mathrm{Hb}]$ was found to be significant in any optode after correction using FDR.

Exploratory post hoc comparisons with sham stimulation were performed for spatial visualization. High-frequency stimulation created clear differences in the left hemisphere around the area of stimulation when compared to sham stimulation [Fig. 3(c)]. Single pulse stimulation appeared to produce small and localized activity near the area of stimulation at optode 1 [Fig. 3(b)], and theta burst stimulation did not appear to elicit significant activity during the time window analyzed [Fig. 3(d)].

Post hoc comparisons of the LME linear hypothesis for the surviving channel showed that high-frequency stimulation produced significantly higher changes in $[\mathrm{HbO}]$ than sham stimulation $(p<0.005)$ in optode 6 . The effect of high-frequency stimulation was also significantly different from that of single pulse $(p=0.04)$ but not significantly different from that of theta burst stimulation. Analysis using Tukey's honestly significant difference comparisons produced similar results with significant differences for high frequency with sham $(p<0.0001)$ and single pulse stimulation ( $p=0.013)$. The temporal changes and mean changes in optode 6 associated with each stimulation type are presented in Figs. 4 and 5, respectively. Changes in [Hb] levels were not observed to be significant for any stimulation type in optode 6 . 
(a)

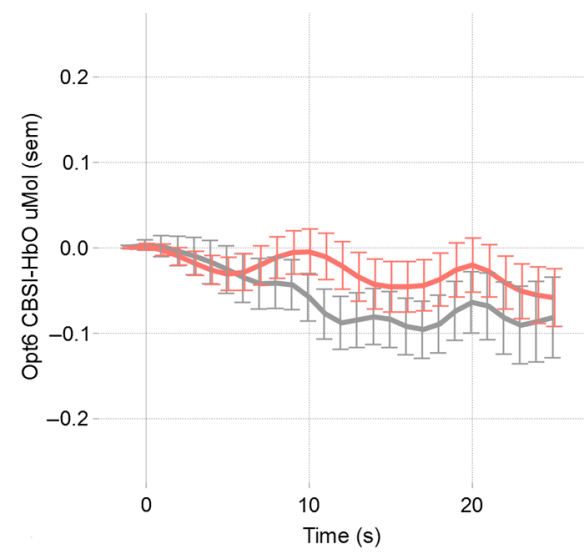

(b)

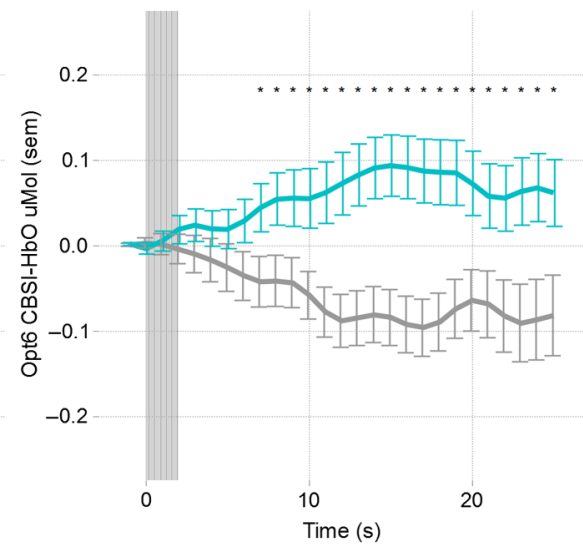

(c)

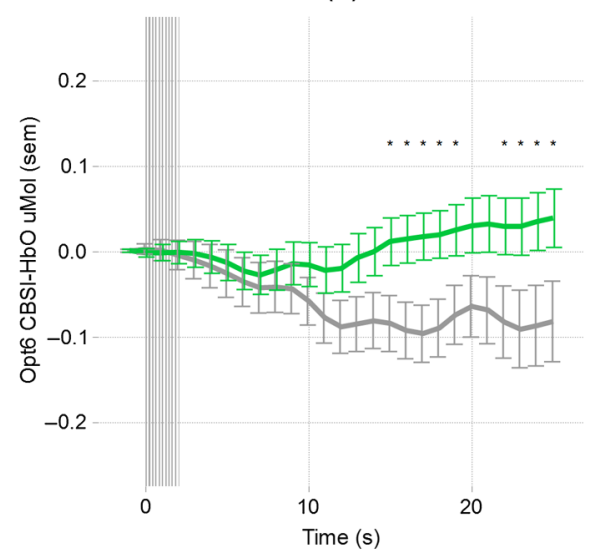

Fig. 4 Averaged response across all subjects following (a) single pulse, (b) high frequency, and (c) theta burst stimulation, respectively, for optode 6 , the gray curves denote the case of sham stimulation.

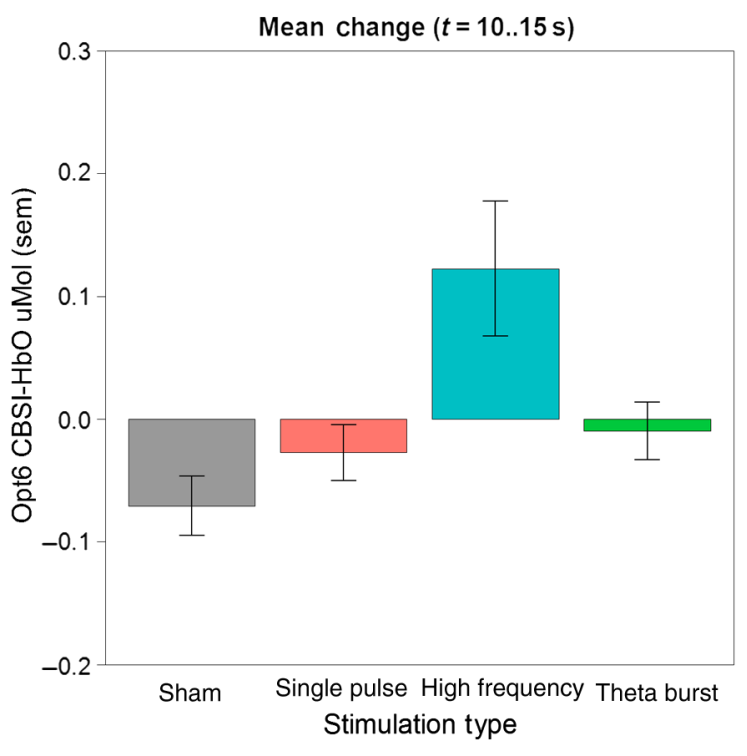

Fig. 5 Mean change (10 to $15 \mathrm{~s}$ ) from baseline across all subjects following single pulse, high frequency, and theta burst stimulation, respectively, for optode 6 .

\section{Discussion}

In this study, we monitored the functional hemodynamic response in the DLPFC during and immediately following different types of patterned stimulation applied at F3. We observed a clear evoked response to the $15-\mathrm{Hz}$ stimulation at $110 \%$ RMT. The evoked response was most strongly present in the brain area close to the stimulation (i.e., optode 6). We observed that the single pulse condition and the 2-s theta burst condition produced minimally apparent activity in areas that were localized near the site of stimulation (optode 1 and 2, respectively), but these changes were marginal compared with the responses produced by high-frequency stimulation and did not survive the significance test after correction for multiple comparisons. Our results suggest that: (i) responses to suprathreshold high-frequency stimulation can be safely monitored using LED-based NIRS systems placed proximally to the site of stimulation and (ii) subthreshold theta burst stimulation and suprathreshold single pulse stimulation do not appear to produce reliable responses at locations adjacent to the coil in the DLPFC.

Our primary finding is that short trains of suprathreshold high-frequency stimulation produce a localized response in the PFC. This finding supports a previous observation that high-frequency stimulation ( $5 \mathrm{~s}, 5 \mathrm{~Hz}, 110 \%$ RMT) results in an increase in $[\mathrm{HbO}]$ peaking at $\sim 5$ to 10 s poststimulation. ${ }^{37}$ However, this is the first online fNIRS study to report changes using a $15-\mathrm{Hz}$ stimulation frequency as well as the first study to report on the effects of HF stimulation with a duration of only $2 \mathrm{~s}$, in which cortical changes produced from TMS are measured with fNIRS. Previous research has shown that in terms of therapeutic outcome for high-frequency TMS, the total number of pulses is more important than the specific frequency used. ${ }^{23}$ rTMS has been known to produce changes in excitability within the motor cortex for frequencies greater than $2 \mathrm{~Hz}$ after only 20 pulses. ${ }^{38}$ Therefore, we anticipated a detectable response following a stimulation dosage of 30 suprathreshold pulses.

The lack of significant response to single pulse stimulation at $110 \%$ RMT is supported by a previous study of the DLPFC, in which a suprathreshold stimulation of $130 \%$ RMT was required to elicit an observable response, captured as a decrease in $[\mathrm{HbO}] .{ }^{21}$ It is possible that different areas of the brain respond differently to similar stimulation paradigms, as single pulse stimulation at subthreshold intensities (65\% to $100 \%$ RMT) has been associated with increases in $[\mathrm{HbO}]$ as measured by fNIRS in the motor cortex, ${ }^{19,20}$ but conflicting reports have also suggested decreases in [Hb], which have different implications for total hemoglobin in this same region. ${ }^{18}$ While it appeared that in our study, single pulse stimulation at $110 \%$ increased $[\mathrm{HbO}]$ in optode 1 , the changes did not survive correction for multiple hypotheses, suggesting that the response was either variable, or small and transient. Given the conflicting reports regarding SP stimulation in M1, our confirmation of nonsignificant response to suprathreshold stimulation in the PFC demonstrates a need for a comparative study between stimulation in M1 and the PFC as different processes may govern the effects of TMS in these areas.

Our investigation into the online monitoring of cortical changes induced by single trains of iTBS (2-s iTBS, 90\% RMT) is the first online fNIRS study to evaluate iTBS in the DLPFC. We found that seconds of iTBS at $90 \%$ RMT was not associated with reliable evoked responses outside of the 
immediate area of stimulation. We observed that optode 6 , in the left hemisphere near the site of stimulation, was associated with increased levels of [HbO], however, these changes did not survive correction for multiple hypotheses. In this study, highfrequency stimulation at $15 \mathrm{~Hz}$ and iTBS stimulation with equal pulse numbers delivered during a period of $2 \mathrm{~s}$ were used to determine whether a single iTBS stimulation train could produce a comparable response to $15-\mathrm{Hz}$ stimulation despite a subthreshold stimulation level. iTBS has been examined previously in one study that examined stimulation to the premotor cortex, M1, and the sensory motor cortex, but not the PFC. Mochizuki et al. found that iTBS trains reduced [HbO] and that 57\% RMT and $70 \%$ RMT reduced contralateral $[\mathrm{HbO}]$ in each region. ${ }^{39}$ Despite increased stimulus intensity relative to the prior study, reports of superior efficacy to iTBS stimulation in terms of clinical respons $\mathrm{e}^{40}$ and increased sensitivity of motor threshold to the pattern relative to high frequency, ${ }^{3,41}$ we did not observe significant changes in the area adjacent to the site of stimulation at subthreshold intensities. It is possible that the reduction in intensity that is necessary for TBS reduced the focality of the stimulation, as intensity used in TMS controls both the area and depth of activation. ${ }^{15}$ It is also likely that suprathreshold stimulation in the DLPFC is required in order to observe a hemodynamic response to short trains of stimulation perhaps indicating a reduced sensitivity of the DLPFC to TMS stimulation relative to $\mathrm{M} 1$.

Previous fNIRS studies have been conducted with laserbased fNIRS systems, ${ }^{16}$ which typically use optical fibers as light guides for both the detector and emitter components. This technique prevents even the potential influence of TMS magnetic flux from interfering with the fNIRS measurements but requires substantial increase of technical complexity, reducing practical use and requiring a longer setup time. The system used in this study was an LED-based fNIRS system, which was developed with a wearable sensor pad for practical setup and use. In order to establish the feasibility of online TMS measurement with the LED-based system, we examined the electrical activity produced by the TMS coil in the fNIRS sensor with an oscilloscope. Minor transient changes were observed in the sensor wires that lasted for several microseconds when the TMS coil was pressed directly over the sensor at $100 \%$ machine output power. When the sensor was used during online recording, TMS-related stimulation produced a transient impulse $(80 \mu \mathrm{s},<5 \mathrm{mv})$ response lasting one sample, which was observable only in sensor data without the LED on. While interference was expected from the TMS coil, it appears that the relatively low sampling rate of the fNIRS sensor and the marginal impulse detected, together ensured that the induced TMS noise remained at undetectable levels. Changes remained negligible even under conditions of repeated stimulation at levels much higher than those used in the study. Other LEDbased fNIRS systems should be tested to ensure compatibility with TMS systems as the lack of interference in the utilized system cannot be assumed for other sensor designs.

This study is subject to several limitations, the foremost of which is the lack of information regarding the cortical areas subject to the direct and downstream effects of magnetic stimulation that were not measured. TMS-induced changes have been shown to occur at regions remote to the direct area of activation ${ }^{42,43}$ and these links may relate to complex relations in functional connectivity between regions. ${ }^{44}$ Despite the inaccessibility of focal measurements in this methodology, we were able to successfully measure changes associated with suprathreshold high-frequency stimulation with fNIRS. Although the contribution of extracerebral sources to the measured evoked responses were not investigated in this study, in future works the use of alternative sensor geometry arrangements (e.g., short separation source detectors pairs) can be used to mitigate the influence of superficial factors and potentially improve the signal-to-noise ratio of measured cerebral responses. A distinct limitation to the use of fNIRS to measure TMS evoked responses is the relatively shallow depth of fNIRS recording relative to fMRI. However, TMS stimulation itself is relatively shallow, ${ }^{45}$ and while coil designs have been adapted in efforts to increase the depth of stimulation, coils commonly used in the clinic reach depths that are relatively well suited to fNIRS measurement. ${ }^{15}$

Additionally, the choice of stimulation paradigms used is not representative of all stimulation paradigms used within the clinic. We did not investigate low-frequency stimulation as the $1-\mathrm{Hz}$ frequency stimulation for a $2-\mathrm{s}$ stimulation train would consist of only two pulses. However, trains of low-frequency stimulation have been reported to reduce [HbO] in both relatively short trains (5 to $10 \mathrm{~s})^{37,46}$ and over $10 \mathrm{~min}$ of stimulation ${ }^{47}$ in the DLPFC at suprathreshold levels $(110 \%$ to $120 \%$ RMT). With respect to the patterned protocols used in this study, the HF and TBS paradigms were not matched in terms of stimulus intensity in part because suprathreshold TBS stimulation is not currently considered low risk ${ }^{30}$ and in part because subthreshold HF stimulation does not produce localized responses in fMRI. ${ }^{27,28}$ As a result, it was not possible to separate the impact of stimulation pattern from intensity in this study, however, subthreshold application of TMS is known to change CBF over longer time scales than analyzed in this study, ${ }^{11,48,49}$ meaning that absence of an immediate observed response does not prohibit clinical efficacy.

In part because there is no overt output from the DLPFC, the changes in cortical response to stimulation behavior with increased dosages of stimulation are not known. The stimulation doses used in this study were smaller than typical therapeutic doses ( $\leq 300$ pulses) and previous studies of cortical excitability in the motor cortex have suggested that changes induced by larger (600 pulses) were no longer significant at $90 \mathrm{~min}$ following stimulation. ${ }^{50}$ Stimulation sessions occurring on the same day were separated an average washout period of $3.3 \mathrm{~h}$ (s.d. $84 \mathrm{~min}$ ). In general, changes in cognition are estimated to be observable only within half the duration of the rTMS train, ${ }^{51}$ much shorter than the length of time used in this study. In combination with the usage of randomized stimulation orders to prevent order effects, these factors suggested that the washout period was adequate. Additionally, all stimulation paradigms in this study used biphasic stimulation. For single pulse paradigms, the nature of the evoked response to biphasic stimulation is thought to be more complex and nuanced than comparable monophasic stimulation. ${ }^{23}$ Due to the increased energy requirements of monophasic stimulation, rTMS techniques used in the clinic and nonsingle pulse applications are commonly biphasic, and therefore, this mode was used in the presented study.

The combination of noninvasive neuroimaging and neurostimulation methodologies represents a unique opportunity to test neuroscientific theories on cortical function and therapy. ${ }^{52}$ It is unknown precisely what implications an individual's measured response to TMS might have on therapeutic outcomes, but 
interpreting feedback from neurostimulation may empower both diagnostic and therapeutic methods. ${ }^{53}$ Without limiting potential applications to clinical populations, neurostimulation could intersect with the merging of neuroimaging and behavioral sciences and bring new dimensions to research as a set of tools investigating the nature of workload and cognition in the brain during everyday life. ${ }^{54-56}$

In conclusion, we have confirmed findings that short trains of high-frequency stimulation are associated with increases in $[\mathrm{HbO}]$ in the DLPFC and demonstrated the feasibility of monitoring online responses to TMS-evoked activity using an LEDbased fNIRS system. Our results generate further support for speculations that the DLPFC is not as sensitive to stimulation as M1, and therefore, future work is needed to specifically evaluate the differences in TMS effects between these regions. These findings highlight the importance of neuroimaging methods to evaluate TMS effects in areas with no overt response and may have important clinical implications regarding the translation of observed phenomena in M1 to regions used in psychiatric studies such as the DLPFC. Future studies are needed to understand the nature of patterned stimulation in the DLPFC, and how evoked responses are related to clinical outcome.

\section{Disclosures}

Results from this study were presented at the 2016 biennial meeting of the Society for fNIRS.

\section{Acknowledgments}

This work was supported in part by grants from the National Natural Science Foundation of China (No. 61673267), the Natural Science Foundation of Shanghai (No. 16ZR1446600), and Med-X Research Fund of Shanghai Jiao Tong University (No. YG2015ZD12). fNIR Devices, LLC manufactures the optical brain imaging instrument and licensed IP and knowhow from Drexel University. H. Ayaz and B. Onaral were involved in the technology development and thus offered a minor share in the new startup firm fNIR Devices, LLC.

\section{References}

1. N. Nevler and E. L. Ash, "TMS as a tool for examining cognitive processing," Curr. Neurol. Neurosci. Rep. 15(8), 52 (2015).

2. E. M. Wassermann and T. Zimmermann, "Transcranial magnetic brain stimulation: therapeutic promises and scientific gaps," Pharmacol. Ther. 133(1), 98-107, (2012).

3. Y. Z. Huang et al., "Theta burst stimulation of the human motor cortex," Neuron 45(2), 201-206 (2005).

4. L. Cárdenas-Morales et al., "Mechanisms and applications of theta-burst rTMS on the human motor cortex," Brain Topogr. 22(4), 294-306 (2010).

5. A. Suppa et al., "Ten years of theta burst stimulation in humans: established knowledge, unknowns and prospects," Brain Stimul. 9(3), 323-335, (2016).

6. Z. J. Daskalakis and S. Commentary, "Theta-burst transcranial magnetic stimulation in depression: when less may be more," Brain 137(7), 18601862 (2014).

7. T. A. Kimbrell et al., "Frequency dependence of antidepressant response to left prefrontal repetitive transcranial magnetic stimulation (rTMS) as a function of baseline cerebral glucose metabolism," Biol. Psychiatry 46(99), 1603-1613 (1999).

8. V. P. Clark and R. Parasuraman, "Neuroenhancement: enhancing brain and mind in health and in disease," Neuroimage 85, 889-894 (2014).

9. C. K. Loo et al., "High $(15 \mathrm{~Hz})$ and low $(1 \mathrm{~Hz})$ frequency transcranial magnetic stimulation have different acute effects on regional cerebral blood flow in depressed patients," Psychol. Med. 33(6), 997-1006 (2003).
10. A. M. M. Speer et al., "Opposite effects of high and low frequency rTMS on mood in depressed patients: relationship to baseline cerebral activity on PET," J. Affective Disord. 115(3), 386-394 (2009).

11. Z. Nahas et al., "Unilateral left prefrontal transcranial magnetic stimulation (TMS) produces intensity-dependent bilateral effects as measured by interleaved BOLD fMRI," Biol. Psychiatry 50(9), 712-720 (2001).

12. H. R. Siebner et al., "Consensus paper: combining transcranial stimulation with neuroimaging," Brain Stimul. 2, 58-80 (2009).

13. P. Homan et al., "Cerebral blood flow identifies responders to transcranial magnetic stimulation in auditory verbal hallucinations," Transl. Psychiatry 2(11), e189 (2012).

14. H. Ayaz et al., "Continuous monitoring of brain dynamics with functional near infrared spectroscopy as a tool for neuroergonomic research: empirical examples and a technological development," Front. Hum. Neurosci. 7, 871 (2013).

15. A. Thielscher and T. Kammer, "Electric field properties of two commercial figure-8 coils in TMS: calculation of focality and efficiency," Clin. Neurophysiol. 115, 1697-1708 (2004).

16. N. A. Parks, "Concurrent application of TMS and near-infrared optical imaging: methodological considerations and potential artifacts," Front. Hum. Neurosci. 7, 592 (2013).

17. R. McKendrick, R. Parasuraman, and H. Ayaz, "Wearable functional near infrared spectroscopy (fNIRS) and transcranial direct current stimulation (tDCS): expanding vistas for neurocognitive augmentation," Front. Syst. Neurosci. 9, 1-14 (2015).

18. H. Mochizuki et al., "Cortical hemoglobin-concentration changes under the coil induced by single-pulse TMS in humans: a simultaneous recording with near-infrared spectroscopy," Exp. Brain Res. 169(3), 302-310 (2006).

19. Y. Noguchi, E. Watanabe, and K. L. Sakai, "An event-related optical topography study of cortical activation induced by single-pulse transcranial magnetic stimulation," Neuroimage 19, 156-162 (2003).

20. T. Furubayashi et al., "Cortical hemoglobin concentration changes underneath the coil after single-pulse transcranial magnetic stimulation: a near-infrared spectroscopy study," J. Neurophysiol. 109(6), 16261637 (2013).

21. R. H. Thomson et al., "Blood oxygenation changes resulting from suprathreshold transcranial magnetic stimulation," Brain Stimul. 4(3), 165-168 (2011).

22. R. H. Thomson, Z. J. Daskalakis, and P. B. Fitzgerald, "A near infra-red spectroscopy study of the effects of pre-frontal single and paired pulse transcranial magnetic stimulation," Clin. Neurophysiol. 122(2), 378-382, (2011).

23. J.-P. Lefaucheur et al., "Evidence-based guidelines on the therapeutic use of repetitive transcranial magnetic stimulation (rTMS)," Clin. Neurophysiol. 125, 2150-2206 (2014).

24. H. Ayaz et al., "Using MazeSuite and functional near infrared spectroscopy to study learning in spatial navigation," J. Visualized Exp. 56, 3443 (2011).

25. D. Rossini et al., "Transcranial magnetic stimulation in treatmentresistant depressed patients: a double-blind, placebo-controlled trial," Psychiatry Res. 137(1-2), 1-10 (2005).

26. A. A. Gershon, P. N. Dannon, and L. Grunhaus, "Transcranial magnetic stimulation in the treatment of depression," Am. J. Psychiatry 160(5), 835-845 (2003).

27. S. Bestmann et al., "Subthreshold high-frequency TMS of human primary motor cortex modulates interconnected frontal motor areas as detected by interleaved fMRI-TMS," Neuroimage 20(3), 1685-1696 (2003).

28. S. Bestmann et al., "BOLD MRI responses to repetitive TMS over human dorsal premotor cortex," Neuroimage 28(1), 22-29 (2005).

29. S. Bestmann et al., "Functional MRI of the immediate impact of transcranial magnetic stimulation on cortical and subcortical motor circuits," Eur. J. Neurosci. 19(7), 1950-1962 (2004).

30. L. Oberman et al., "Safety of theta burst transcranial magnetic stimulation: a systematic review of the literature," J. Clin. Neurophysiol. 28(1), 67-74 (2011).

31. H. Ayaz et al., "Sliding-window motion artifact rejection for functional near-infrared spectroscopy," Annual Int. Conf. of the IEEE Engineering in Medicine and Biology Society (EMBC '10), pp. 65676570 (2010). 
32. D. T. Delpy et al., "Estimation of optical pathlength through tissue from direct time of flight measurement," Phys. Med. Biol. 33(12), 1433-1442 (1988).

33. X. Cui, S. Bray, and A. L. Reiss, "Functional near infrared spectroscopy (NIRS) signal improvement based on negative correlation between oxygenated and deoxygenated hemoglobin dynamics," Neuroimage 49(4), 3039-3046 (2010).

34. C. R. Genovese, N. A. Lazar, and T. Nichols, "Thresholding of statistical maps in functional neuroimaging using the false discovery rate," Neuroimage 15(4), 870-878 (2002).

35. C. Krueger and L. Tian, "A comparison of the general linear mixed model and repeated measures ANOVA using a dataset with multiple missing data points," Biol. Res. Nurs. 6(2), 151-157 (2004).

36. M. Ferrari and V. Quaresima, "A brief review on the history of human functional near-infrared spectroscopy (fNIRS) development and fields of application," Neuroimage 63(2), 921-935 (2012).

37. T. T. Cao et al., "A near infra-red study of blood oxygenation changes resulting from high and low frequency repetitive transcranial magnetic stimulation," Brain Stimul. 6, 922-924 (2013).

38. A. Pascual-Leone et al., "No evidence of hearing loss in humans due to transcranial magnetic stimulation," Neurology 42 (3 Pt 1), 647 (1992).

39. H. Mochizuki et al., "Hemoglobin concentration changes in the contralateral hemisphere during and after theta burst stimulation of the human sensorimotor cortices," Exp. Brain Res. 180(4), 667-675 (2007).

40. C. T. Li et al., "Efficacy of prefrontal theta-burst stimulation in refractory depression: a randomized sham-controlled study," Brain 137(7), 2088-2098 (2014).

41. Y.-Z. Huang et al., "The theoretical model of theta burst form of repetitive transcranial magnetic stimulation," Clin. Neurophysiol. 122(5), 1011-1018 (2011).

42. Y. Aoyama et al., "Stimulus intensity dependence of cerebral blood volume changes in left frontal lobe by low-frequency rTMS to right frontal lobe: a near-infrared spectroscopy study," Neurosci. Res. 63, 47-51 (2009).

43. M. D. Fox et al., "Measuring and manipulating brain connectivity with resting state functional connectivity magnetic resonance imaging (fcMRI) and transcranial magnetic stimulation (TMS)," Neuroimage 62(4), 2232-2243 (2012).

44. F. A. Kozel et al., "Using simultaneous repetitive transcranial magnetic stimulation/functional near infrared spectroscopy (rTMS/fNIRS) to measure brain activation and connectivity," Neuroimage 47(4), 11771184 (2009).
45. Z. De Deng, S. H. Lisanby, and A. V. Peterchev, "Electric field depthfocality tradeoff in transcranial magnetic stimulation: simulation comparison of 50 coil designs," Brain Stimul. 6(1), 1-13 (2013).

46. F. Tian et al., "Test-retest assessment of cortical activation induced by repetitive transcranial magnetic stimulation with brain atlas-guided optical topography," J. Biomed. Opt. 17(11), 116020 (2012).

47. R. H. Thomson et al., "Intensity dependent repetitive transcranial magnetic stimulation modulation of blood oxygenation," J. Affective Disord. 136(3), 1243-1246 (2012).

48. A. Orosz et al., "Theta burst TMS increases cerebral blood flow in the primary motor cortex during motor performance as assessed by arterial spin labeling (ASL)," Neuroimage 61(3), 599-605 (2012).

49. A. M. Speer et al., "Intensity-dependent regional cerebral blood flow during $1-\mathrm{Hz}$ repetitive transcranial magnetic stimulation (rTMS) in healthy volunteers studied with $\mathrm{H} 215 \mathrm{O}$ positron emission tomography: I. Effects of primary motor cortex rTMS," Biol. Psychiatry 54(8), 818825 (2003).

50. M. Wischnewski and D. J. L. G. Schutter, "Efficacy and time course of theta burst stimulation in healthy humans," Brain Stimul. 8(4), 685-692 (2015).

51. E. M. Robertson, H. Théoret, and A. Pascual-Leone, "Studies in cognition: the problems solved and created by transcranial magnetic stimulation," J. Cogn. Neurosci. 15(7), 948-960 (2003).

52. W. Teo et al., "Does a combination of virtual reality, neuromodulation and neuroimaging provide a comprehensive platform for neurorehabilitation?-a narrative review of the literature," Front. Hum. Neurosci. 10, 1-15 (2016).

53. D. Guhathakurta and A. Dutta, "Computational pipeline for NIRS-EEG joint imaging of tDCS-evoked cerebral responses-an application in ischemic stroke," Front. Neurosci. 10, 261 (2016).

54. G. Derosière et al., "NIRS-measured prefrontal cortex activity in neuroergonomics: strengths and weaknesses," Front. Hum. Neurosci. 7, 1-3 (2013).

55. K. Mandrick et al., "Why a comprehensive understanding of mental workload through the measurement of neurovascular coupling is a key issue for neuroergonomics?" Front. Hum. Neurosci. 10, 1-5 (2016).

56. S. Perrey, "Non-invasive NIR spectroscopy of human brain function during exercise," Methods 45(4), 289-299 (2008).

Biographies for the authors are not available. 